\title{
Obfuscation by Partial Evaluation of Distorted Interpreters
}

\author{
Neil D. Jones \\ DIKU, University of Copenhagen (prof. emeritus) \\ Joint work with Roberto Giacobazzi and Isabella Mastroeni \\ University of Verona \\ Paper: in PEPM 2012
}


Obfuscation = hiding of intended meaning in communication, making a program confusing, wilfully ambiguous, and harder to interpret.

- Making something dark

- Putting something in a shadow

Our version: a semantics-preserving program transformation intended to make transformed programs hard to understand.

Popular in the computer security and software engineering communities: Obfuscation, Watermarking, Steganography. WHY?

- To avoid theft of an algorithm (hard to steal or adapt or patent)

to hide evidence in a program of its authorship, ownership, creator

- ... related other security techniques... 
A scenario:

- An attacker is trying to analyze or decipher obfuscated program $\mathrm{p}^{\prime}$

- a defender is trying to construct $\mathrm{p}^{\prime}$ to make this hard to do

Want: $\mathrm{p}^{\prime}$ is executable, but it should be hard to adapt, exploit, or analyze.

SOME CRITERIA:

1. Semantics preservation: we must have

$$
\forall p \cdot \llbracket p^{\prime} \rrbracket=\llbracket p \rrbracket
$$

2. Automation: $\mathrm{p}^{\prime}$ is obtained from $\mathrm{p}$ without hand work.

Thus the programmer/inventor of $p$ can release $p^{\prime}$ instead of $p$.

3. Efficiency: $\mathrm{p}^{\prime}$ should not be too much slower or larger than $\mathrm{p}$.

4. Potency $=$ hard reverse engineering, namely

$\mathrm{p}$ is hard to obtain from its obfuscated version $\mathrm{p}^{\prime}$. 


\section{HOW ? OUR SILVER BULLET}

Program transformation by specializing a self-interpreter.

Program interp is a self-interpreter if for all programs $\mathrm{p}$ and data $\mathrm{d} \in \mathbb{D}$

$$
\llbracket p \rrbracket(d)=\llbracket i n t e r p \rrbracket(p, d)
$$

A partial evaluator (or program specializer) spec satisifies for every program $p$ with "static" input $s \in \mathbb{D}$ and "dynamic" input $d \in \mathbb{D}$, that

$$
\llbracket p \rrbracket(s, d)=\llbracket \llbracket \text { spec } \rrbracket(p, s) \rrbracket(d)
$$

Some practical program specializers:

Tempo and CMix for C; ECCE and Logen for Prolog; Unmix, Similix and PGG for SCHEME.

We used Unmix in our experiments. 


\section{PROGRAM TRANSFORMATION BY INTERPRETER SPECIALIZATION}

Suppose

$$
\mathrm{p}^{\prime}:=\llbracket \mathrm{spec} \rrbracket(\text { interp, } \mathrm{p})
$$

So $\mathrm{p}^{\prime}$ is the result of specializing a self-interpreter to program $\mathrm{p}$.

Claim: $\llbracket p \rrbracket=\llbracket p^{\prime} \rrbracket$, by simple equational reasoning. For any data $d$,

$$
\begin{array}{rlrl}
\llbracket p \rrbracket(d) & =\llbracket i n t e r p \rrbracket(p, d) & & \text { definition of self-interpreter } \\
& =\llbracket[\text { spec }](\text { interp, } p) \rrbracket(d) & \text { definition of specializer } \\
& =\llbracket p^{\prime} \rrbracket(d) & & \text { definition of } p^{\prime}
\end{array}
$$

Therefore the function

$$
\mathrm{p} \mapsto \llbracket \text { spec } \rrbracket(\text { interp }, \mathrm{p})
$$

is a semantics-preserving program transformer. 
In the transformation

$$
\mathrm{p} \mapsto \mathrm{p}^{\prime}=\llbracket \text { spec } \rrbracket(\text { interp, } \mathrm{p})
$$

For optimal specialization:

But... for good obfuscation:

$p^{\prime}$ should be as efficient as $p$. $\mathrm{p}^{\prime}$ should be harder to understand than $\mathrm{p}$.

Conflicting goals, but achievable by (re-)designing interp cleverly.

We show that several useful program obfuscations can be obtained by interpreter specialization.

A bit of useful slack:

It is OK for interp to be slow, as long as

$\mathrm{p}^{\prime}$ is fast enough, and hard enough to understand. 


\section{IN GENERAL, IF p' $=\llbracket$ spec $\rrbracket($ interp, $p)$ :}

1. Program $\mathrm{p}^{\prime}$ inherits the algorithm of program $\mathrm{p}$.

2. Program $p^{\prime}$ inherits the programming style of interp.

Our trick: build a program transformer

- by programming a self-interpreter interp ${ }^{+}$

$\checkmark$ in a style to give the desired transformation.

- Then (automatically)

- specialise interp ${ }^{+}$to any input program $\mathrm{p}$

- to transform $\mathrm{p}$ as desired.

Some writing styles that can be inherited from interp:

- functional language, tail-recursive ,or CPS (continuation-passing) styles

- Or interp can use memoisation to implement function calls. 


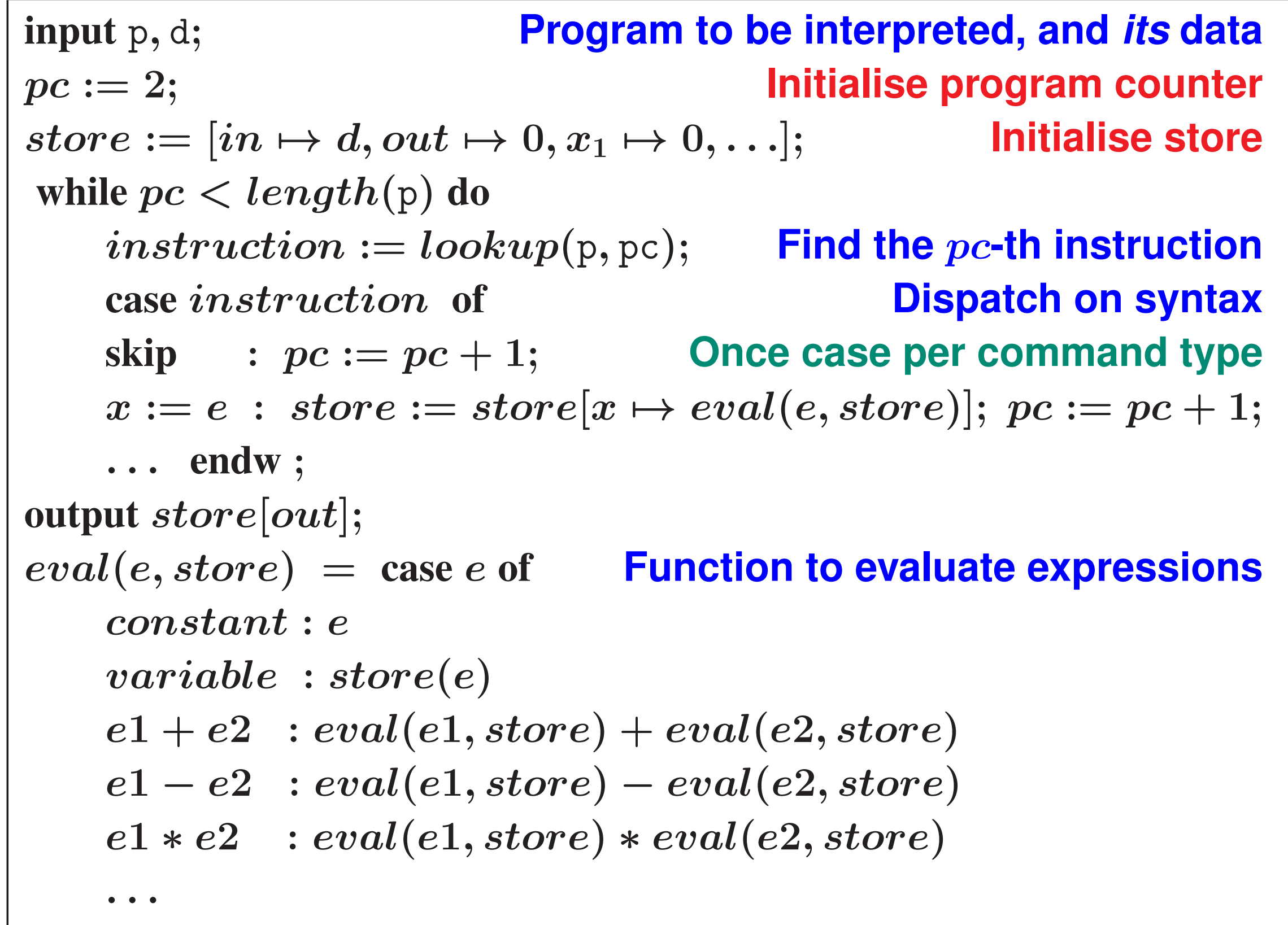


A simple color-coding scheme:

- GREEN for static(ally computable)

- RED for dynamic.

First steps using this coding:

- interp variable $\mathrm{p}$ is classified as "static", and variable $d$ is classified as "dynamic".

-... and a bit more:

- interp variables $e, p c$ and instruction are classified as "static", since given any $p$ they can only assume finitely many values. 


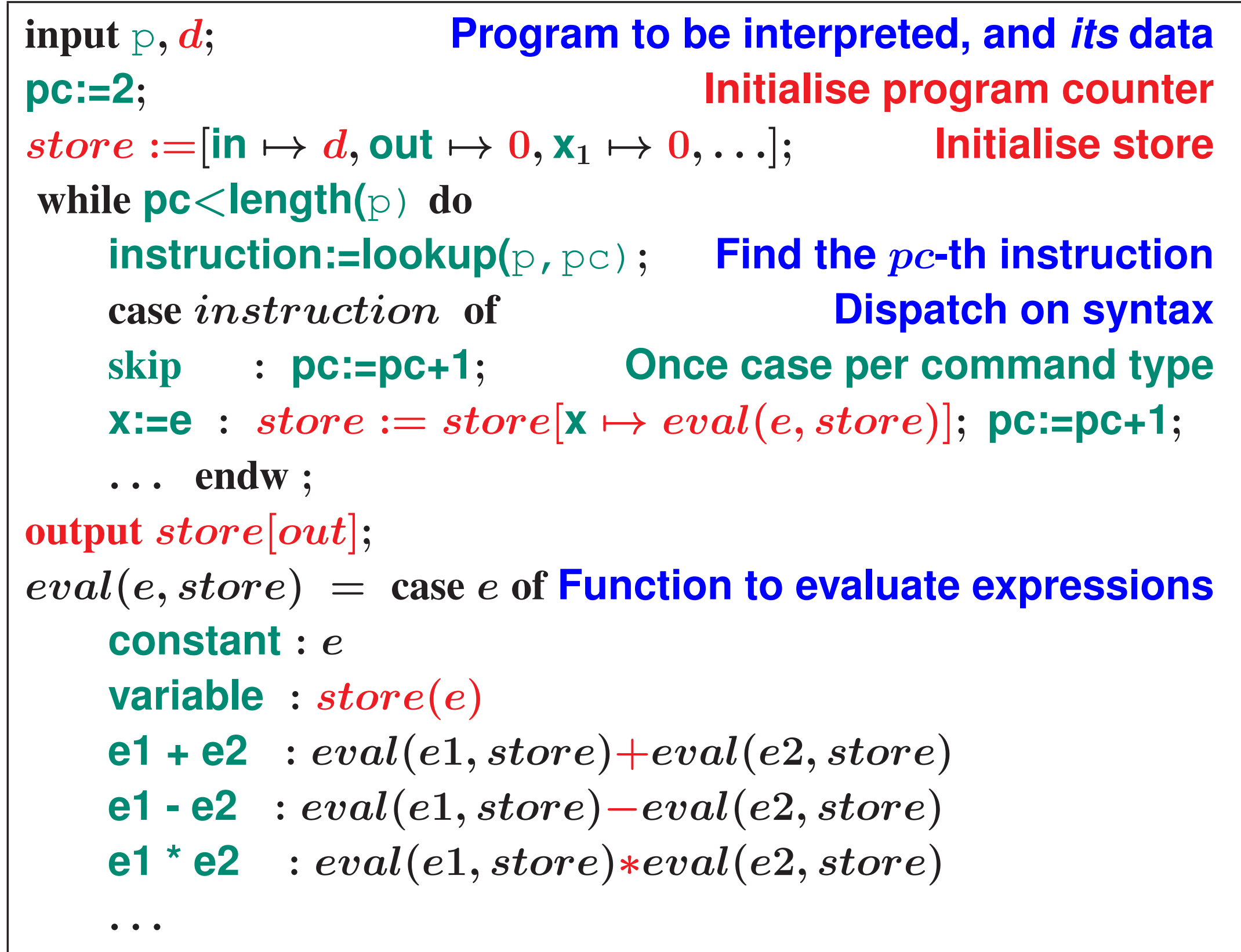




\section{SPECIALIZATION OF THE SIMPLE SELF-INTERPRETER}

D interp variable $\mathrm{p}$ is classified as "static", and variable $d$ is classified as "dynamic".

- interp variables $e, p c$ and instruction are classified as "static", since given any $\mathrm{p}$ they can only assume finitely many values.

- The interpreter's while loop is unfolded, so the only remaining control transfers implement the transfers in program $\mathrm{p}$. 


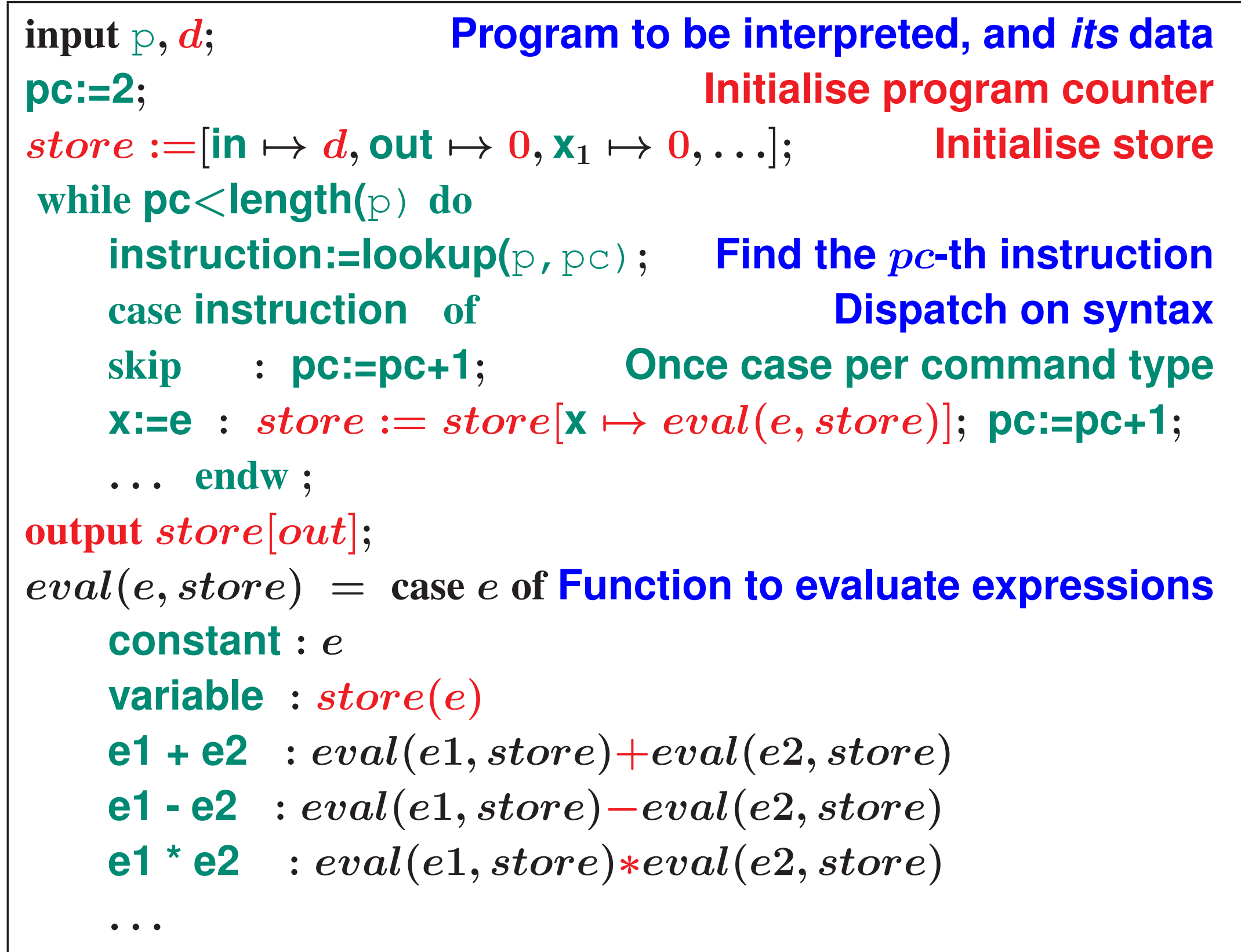




\section{SPECIALIZATION OF THE SIMPLE SELF-INTERPRETER}

D interp variable $\mathrm{p}$ is classified as "static", and variable $d$ is classified as "dynamic".

- interp variables $e, p c$ and instruction are classified as "static", since given any $p$ they can only assume finitely many values.

- The interpreter's while loop is unfolded, so the only remaining control transfers correspond to those present in program $\mathrm{p}$.

- interp function eval is completely unfolded and so does not appear in $\mathrm{p}^{\prime}$, since all recursive calls decrease the static value $e$. 


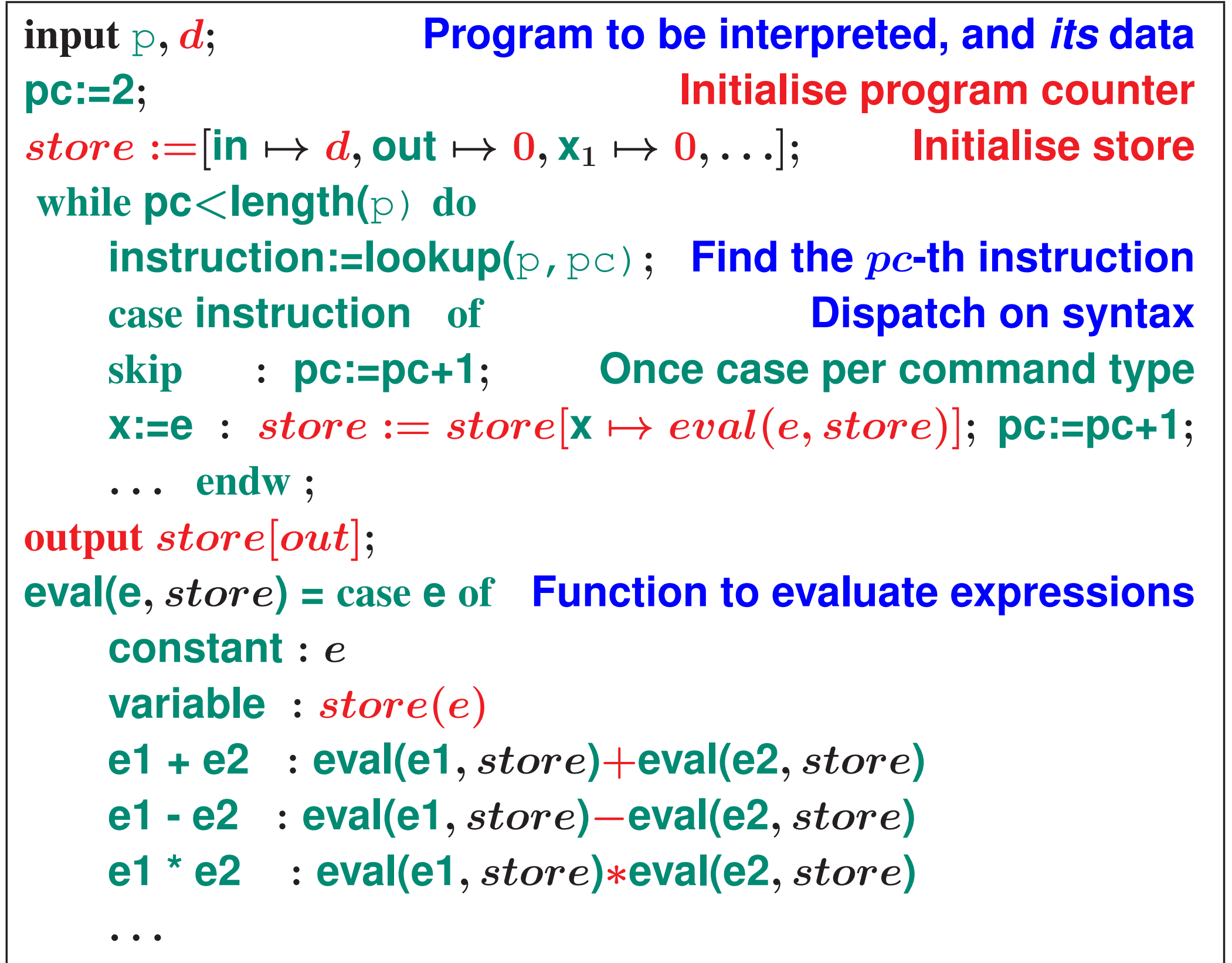




\section{SPECIALIZATION OF THE SIMPLE SELF-INTERPRETER}

- interp variable $\mathrm{p}$ is classified as "static", and variable $d$ is classified as "dynamic".

- interp variables $e, p c$ and instruction are classified as "static", since given any $\mathrm{p}$ they can only assume finitely many values.

- The interpreter's while loop is unfolded, so the only remaining control transfers correspond to those present in program $\mathrm{p}$.

- interp function eval is completely unfolded and so does not appear in $\mathrm{p}^{\prime}$, since all recursive calls decrease the static value $e$.

- interp variable store is a function with static domain but dynamic range.

- "Arity raising" splits store into: one specialised program variable for each of $p$ 's variables. 


\section{SPECIALIZATION OF THE SIMPLE SELF-INTERPRETER}

Net effect of all of these tricks:

- the specialized program $\mathrm{p}^{\prime}=\llbracket \operatorname{spec} \rrbracket($ interp, $\mathrm{p})$

- is identical to $p$ (up to variable renaming).

Alas, this is not what we want for obfuscation

since $\mathrm{p}^{\prime}$ is $\alpha$-equivalent to $\mathrm{p}$

(identical up to renaming $=$ too easy to deobfuscate...)

But it is a first step towards

more interesting automatic program transformation 


\section{AN EASY EXAMPLE: DATA OBFUSCATION}

Data obfuscation

Similar to Drape's technique, but automated.

Modify the simple self-interpreter so that

- all values in the store are obfuscated, e.g., by multiplying by 2 .

- Mutual inverse functions $o b f(x)$ and $\operatorname{dob}(x)$.

Modify interp so that:

- All stored values are obfuscated;

- Input values are obfuscated in the initial store;

- variable values are obfuscated just before putting in the store; and

- output values are de-obfuscated in the program's final store.

- Expression evaluation yields obfuscated values: 
input $\mathrm{p}, \mathrm{d}$;

$p c:=2$;

store $:=[$ in $\mapsto$ obf $(d)$, o
while $p c<$ length $(\mathrm{p})$ do

instruction $:=\operatorname{lookup}(\mathrm{p}, \mathrm{pc})$;

case instruction of

skip $\quad: p c:=p c+1$;

Program to be interpreted, and its data

$\mathrm{X}:=\mathrm{e}: \operatorname{store}:=\operatorname{store}[x \mapsto \operatorname{obf}(\operatorname{eval}(e$, store $))] ; p c:=p c+1$;

... endw ;

output dob(store $[$ out $]$ );

$\operatorname{obf}(V)=2 * V ; \operatorname{dob}(V)=V / 2$

Obfuscation/de-obfuscation eval $(e$, store $)=$ case $e$ of

constant : obf $(e)$

variable : store $(e)$

Variable values

$\mathrm{e} 1+\mathrm{e} 2$ : obf(dob $($ eval $(e 1$, store $))+\operatorname{dob}($ eval $(e 2$, store $)))$

e1-e2 : obf(dob $($ eval $(e 1$, store $))-\operatorname{dob}(\operatorname{eval}(e 2$, store $)))$ 


\section{Program $\mathrm{p}$}

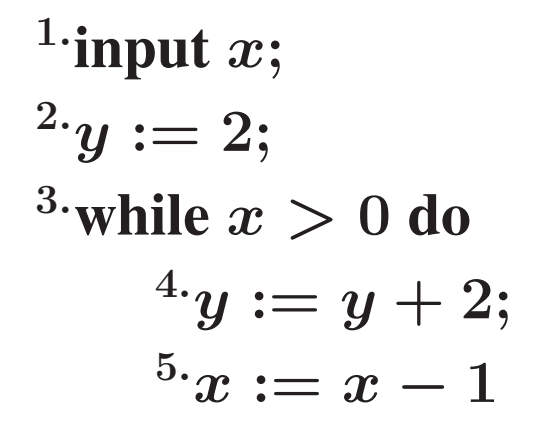

endw

${ }^{6 .}$ output $y$;

7.end

is automatically transformed into this equivalent obfuscated program $\mathrm{p}^{\prime}$ :

${ }^{1}$ input $x$;

1.5. $x:=2 * x ; \quad$ Obfuscate input $x$

${ }^{2 \cdot} y:=2 * 2 ; \quad$ Obfuscate $y:=2$

${ }^{3}$ while $x / 2>0$ do De-obfuscate $x$

${ }^{4 \cdot} y:=2 *(y / 2+2)$;

${ }^{5} \cdot x:=2 *(x / 2-1)$

endw

${ }^{6}$.output $y / 2$; De-obfuscate output

7.end 


\section{WHAT IS HAPPENING ? HOW TO GENERALISE ?}

1. The above example applies one-to-one functions

$$
\text { Value } \frac{\lambda x \cdot 2 x}{\lambda x \cdot x / 2} \text { Value }
$$

2. More generally: apply one-to-one functions

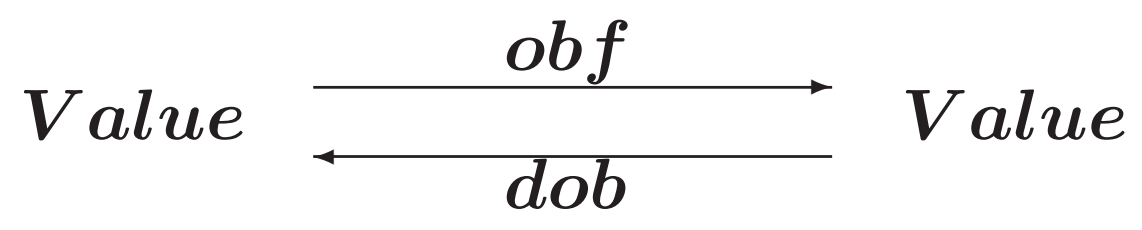

3. Still more generally: apply one-to-one store transformations:

$$
\text { Store } \underset{\text { Stob }}{\longleftarrow} \text { Store }
$$

A nasty example:

$$
\begin{gathered}
o b f(x, y)=(x+y, x-y) \\
\operatorname{dob}(u, v)=((u+v) / 2,(u-v) / 2)
\end{gathered}
$$

This mixes up values of different variables! 


\section{Program $\mathrm{p}$}

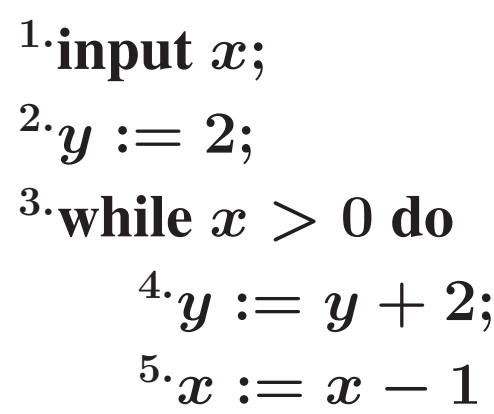

endw

6. output $y$;

$7 \cdot$ end

is automatically transformed into this equivalent obfuscated program $\mathrm{p}^{\prime}$ :

${ }^{1}$ input $x ; u:=x+y ; v:=x-y ; \quad$ Initialise

${ }^{2.1} \cdot u:=(u+v) / 2+2 ; \quad$ Obfuscated $y:=2$

${ }^{2.2 \cdot} v:=(u+v) / 2-2 \quad$-continued-

${ }^{3 .}$ while $(u+v) / 2>0$ do De-obfuscated $x>0$

${ }^{4} \cdot u:=u+2 ; v:=v-2$;

${ }^{5} u:=u-1 ; v:=v-1$;

endw

6. output $(u-v) / 2 ; \quad$ De-obfuscate output

$7 \cdot$ end 


\section{OBFUSCATION FROM A "WHOLE-PROGRAM" VIEWPOINT}

A conflict that makes program obfuscation a subtle problem concerns a general principle in programming:

Good programs are well-structured and have concise invariants.

This is a key to

- understanding a program, and

- adapting it to new purposes.

Good structure and short invariants are necessity in order to develop, debug and perfect a program $\mathrm{p}$ in the first place.

However, instead an obfuscated program

should not be well-structured and should not be easy to understand.

This suggests (among other things):

obfuscation by making the program's control flow hard to understand. 


\section{EXAMPLE OF CODE FLATTENING}

The following flattened program $\mathrm{p}^{\prime}$ has

- only one loop (regardless of how many loops $p$ has), and

- an explicit program counter $p c$

What has been obfuscated?

Original program $\mathrm{p}$ : Flattened equivalent program $\mathrm{p}^{\prime}$ :

${ }^{1}$ input $x$;

${ }^{2 \cdot} y:=2$

${ }^{3}$ while $x>0$ do

${ }^{4 \cdot} y:=y+2$

${ }^{5 \cdot} x:=x-1$

${ }^{1}$ input $x ;{ }^{2} p c:=2$;

${ }^{3}$ while $p c<6$ do

${ }^{4}$ case $p c$ of

$2:{ }^{5 \cdot} y:=2 ;{ }^{6} p c:=3$

$3:{ }^{7}$ if $x>0$ then ${ }^{8 \cdot} p c:=4$ else ${ }^{9} p c:=6$;

$4:{ }^{10} y:=y+2 ;{ }^{11} p c:=5$

endw

$5:{ }^{12 \cdot} x:=x-1 ;{ }^{13} p c:=3$;

${ }^{6}$. output $y$;

7.end

\section{endw}

14. output $y$

${ }^{15}$ end 
Essential trick: to recode interp so that the specializer will classify variable $p c$ as dynamic. Technically

- $p c$ is made dynamic using the Unmix generalize annotation.

- interp is extended to have both dynamic and static copies of $p c$, so specialization will generate $\mathrm{p}^{\prime}$ code such as

$$
\text { case } \ldots p c=5: x:=x-1 ; p c:=3
$$

- Since $p c$ is dynamic, the while loop in interp ${ }^{\text {flat }}$ will not be unfolded, and so $p c$ comes to appear in the specialized program $\mathrm{p}^{\prime}$.

The transformation

$$
\mathrm{p} \mapsto \mathrm{p}^{\prime}=\llbracket \text { spec } \rrbracket\left(\text { interp } \mathrm{p}^{\mathrm{flat}}, \mathrm{p}\right)
$$

will flatten any program; i.e., it is in no way specific to the example program $\mathrm{p}$ above. 
- Ideally, the complexity of obtaining $\mathrm{p}$ from $\mathrm{p}^{\prime}$ should be high (for example, NP-hard).

View: the attacker is an arbitrary PTIME program.

We don't know how to do this (one-way functions... ).

So we settle for an easier solution:

- Obfuscate so that $\mathrm{p}^{\prime}$ is hard to abstractly interpret.

View: the attacker is a program analyzer, e.g., as used in a compiler.

We know better how to do this:

- Use the concept of complete abstract interpretation

(studied by Giacobazzi et al)

- Design self-interpreter interp so that the abstract interpretation of $p^{\prime}$ is not complete (whether or not analysis of $p$ would be complete) 


\section{SIGN ANALYSIS: COMPLETENESS AND INCOMPLETENESS}

Abstract lattice for sign analysis: $\{\perp,+, 0,-, \top\}$

Sign analysis is complete for multiplication $*$ : exact analysis information.

$$
\begin{array}{c|ccc}
* & - & 0 & + \\
\hline- & + & 0 & - \\
0 & 0 & 0 & 0 \\
+ & - & 0 & +
\end{array}
$$

Sign analysis is incomplete for addition +: imprecise analysis inform' $n$.

$$
\begin{array}{c|ccc}
+ & - & 0 & + \\
\hline- & - & - & \top(!) \\
\mathbf{0} & - & \mathbf{0} & + \\
+ & \top(!) & + & +
\end{array}
$$

Our trick: let the interpreter evaluate $*$ using +

$$
\begin{aligned}
& \operatorname{eval}(e, \text { store })=\text { case } e \text { of } \\
& \mathrm{e} 1+\mathrm{e} 2 \text { : eval(e1, store })+\operatorname{eval}(e 2, \text { store }) \\
& \mathrm{e} 1{ }^{*} \mathrm{e} 2 \quad \text { : let } v 1=\operatorname{eval}(e 1, \text { store }), v 2=\operatorname{eval}(e 2, \text { store }) \\
& \text { in } v 1 *(v 2-1)+v 1 \quad \text { The }+ \text { makes analysis imprecise! }
\end{aligned}
$$




\section{OBFUSCATION BY EXPLOITING INCOMPLETENESS}

Program $\mathrm{p}$

Sign analysis of $\mathrm{p}$ yields $y \mapsto+$ in

1.input $x$;

${ }^{2} \cdot y:=2$;

${ }^{3 .}$ while $x>0$ do

${ }^{4 .} \boldsymbol{y}:=\boldsymbol{y} * \boldsymbol{y}$

${ }^{5 \cdot} x:=x-1$

endw

6. output $y$;

$7 \cdot$ end

is automatically transformed into this equivalent obfuscated program $\mathrm{p}^{\prime}$ :

1.input $x$;

${ }^{2} \cdot y:=2$;

${ }^{3 .}$ while $x>0$ do

4. $y:=y *(y-1)+y$;

${ }^{5 \cdot} x:=x-1$

endw

6. output $y$;

7 .end

Sign analysis of $\mathrm{p}^{\prime}$ yields $y \mapsto \top$. 
In the paper, we show the control flow flattening-based obfuscation can be modeled as making incomplete an abstract interpretation.

The attacker is an abstract interpretation extracting the program's control flow graph.

CFG extraction be done several abstraction steps:

- In input we lose the control flow when the program counter is dynamic, namely when it is controlled in the program itself,

- In output we lose the memory and the history of computations (traversed branches).

- An enriched concrete semantics yields a flow chart modeling the history of the computation.

- An abstraction of this concrete semantics yields a flow chart for any program.

Result: this (natural) abstract interpretation is incomplete for a flattened program. 


\section{META-LEVEL DISCUSSION}

The Futamura projections are as follow for a distorted interpreter interp ${ }^{+}$:

1. $\mathrm{p}^{\prime} \quad:=\llbracket$ spec $\rrbracket\left(\right.$ interp $\left.{ }^{+}, \mathrm{p}\right) \quad$ Obfuscate one program

2. comp $:=\llbracket \operatorname{spec} \rrbracket\left(\right.$ spec, interp $\left.{ }^{+}\right)$Generate a stand-alone obfuscator

3. cogen := $=$ spec $\rrbracket($ spec, spec) $\quad$ A generator of obfuscators

The example obfuscating transformations we have seen are instances of the 1st Futamura projection.

2nd Futamura projection: if $\mathrm{P}$ is interp ${ }^{f l a t}$, then compiler is a stand-alone obfuscator: a "flattening" program transformer.

We have done all three using the UNMIX specializer. 


\section{IMMEDIATE CONSEQUENCES OF FUTAMURA PROJ'S}

There are other better ways to obfuscate and to produce a obfuscator:

$\checkmark \mathrm{p}^{\prime}=\llbracket \operatorname{comp} \rrbracket(\mathrm{p})$ (obfuscate by compiler) and

- comp $=\llbracket \operatorname{cogen} \rrbracket\left(\right.$ interp $\left.{ }^{+}\right)$(generate obfuscator).

Future developments will involve gaining a deeper understanding in expected time factors: the relations among

1. time $_{\mathrm{p}^{\prime}}(\mathrm{d})$ and time $_{\mathrm{p}}(\mathrm{d})$ : the slowdown imposed by the obfuscation;

2. time $_{\text {spec }}\left(\right.$ interp $\left.^{+}, \mathrm{p}\right)$ and length $(\mathrm{p})$ : the amount of time to do the obfuscation by general specialization;

3. $\operatorname{time}_{\mathrm{comp}}(\mathrm{p})$ and $\operatorname{length}(\mathrm{p})$ : the amount of time to do the obfuscation by running a generated obfuscator (significantly less than above). 


\section{ANOTHER TYPE OF OBFUSCATION}

A conflict that makes program obfuscation a subtle problem concerns a general principle in programming:

Good programs are well-structured and have concise invariants.

This led to flattening, to:

obfuscation by making the program's control flow hard to understand.

There is another direction to attack the problem:

obfuscation by making the program's invariants and data flow hard to understand.

A next step: insertion of opaque predicates . Trick: replace command C by

$$
\text { if always-false-test then junk-commands else C }
$$

Point: the inserted test and then-branch code will (barely) affect the program execution. But they will complicate life for a program attacker that does not know the program's semantics. 
1. Nice (from my viewpoint): program obfuscation provides a motive to do automatic program transformation

2. Different criteria for success from traditional program transformation:

- not for increased efficiency,

- not to compile, i.e., to change the programming language;

- but to make programs hard to understand or adapt by other people (or by their automated program attack systems)

3. Partial evaluation provides a well-developed approach to do automatic program transformation

4. Starting point: a "vanilla" self-interpreter for the source language

5. This is then distorted, so its specialization to a clear-text source program will produce a

computationally equivalent and correct

but harder-to-understand target program.

6. Examples: data distortion; flattening; inserting opaque predicates. 
S. Chow, Y. Gu, H. Johnson, and V. A. Zakharov. An approach to the obfuscation of control-flow of sequential computer programs. In ISC '01, pp. 144-155. Springer, 2001.

C. Collberg and J. Nagra. Surreptitious Software: Obfuscation, Watermarking, and Tamperproofing for Software Protection. Addison-Wesley Professional, 2009. ISBN 0321549252.

C. Collberg, C. D. Thomborson, and D. Low. Manufacturing cheap, resilient, and stealthy opaque constructs. In 25st ACM POPL '98, pp. 184-196. 1998.

S. Drape. Obfuscation of Abstract Data-Types. PhD thesis, University of Oxford, 2004.

R. Giacobazzi, F. Ranzato, and F. Scozzari. Making abstract interpretation complete. J. of the ACM, 47(2):361-416, March 2000.

N. D. Jones. Transformation by interpreter specialisation. Science of Computer Programming, 52(17(1)):307-339, 2004.

C. Wang, J. Davidson, J. Hill, and J. Knight. Protection of software-based survivability mechanisms. IEEE Int. Conf. Dependable Systems and Networks, pp. 193-202, 2001. 
